\title{
Immunohistochemical localization of NPY, VIP and 5-HT in the thyroid gland of the lizard, Podarcis sicula
}

\author{
R. Sciarrillo1, V. Laforgia, F. Virgilio, R. Longobardi, A. Cavagnuolo, and L. Varano
}

Dipartimento di Biologia Evolutiva e Comparata, Università degli Studi di Napoli "Federico II", Via Mezzocannone 8, 80134 Napoli, Italia and 'Facoltà di Scienze MM.FF.NN., Università degli Studi del Sannio, Via Port'Arsa 11, 82100 Benevento, Italia

Accepted: 31/5/01

Key words: thyroid gland, immunohistochemistry, lizard, NPY, VIP, 5-HT

\section{SUMMARY}

The thyroid gland of the lizard Podarcis sicula was immunohistochemically studied in adult male specimens using specific antibodies against NPY, VIP and 5-HT and the avidin-biotin peroxidase complex $(\mathrm{ABC})$ procedure to localize the three peptides. Fine beaded VIP-immunoreactive nerve fibers ran between the follicles, and VIP-immunoreactivity was evenly distributed in the apical cytoplasm of follicular cells. NPY-immunoreactive fibers were found around the follicles, and, in the cells, immunoreactivity was localizated only in the cellular apices. Immunoreactivity to 5-HT was observed in the colloid, with a concentration in the follicular lumen exceeding that in the follicular cells. In fact, most follicles showed immunoreactivity in the cytoplasmic bridges formed between the apical portion of the follicular cells and the colloid.

\section{INTRODUCTION}

Thyroid function is primarily controlled by pituitary thyroid-stimulating hormone (TSH), but the participation of neuronal factors in this regulation has long been suggested as well. The recent findings that various peptides constitute an integral part of neuronal signal transfer has led to a renewed interest in the physiological role of thyroidal nerves and their products in the regulation of thyroid function.

Thus far, several types of peptide-containing nerve fibers have been observed in the thyroid gland of several species, storing immunoreactive (IR) vasoactive intestinal polypeptide (VIP), neuropeptide Y (NPY) and serotonin (5-HT).

NPY-IR nerve fibers are widely distributed in the body (Sundler et al., 1983; Lundberg et al., 1982). They occur around blood vessels, small arteries and arterioles in particular, in nonvascolar smooth muscle, and around the acini of exocrine glands. Reportedly, NPY reduces local blood flow and enhances adrenergic vasocontriction in vitro (Ekblad et al., 1984). In particular, numerous NPY-IR nerve fibers have been observed around blood vessels, and a moderate number between follicles in the thyroid gland of the species so far studied (mouse, rat, guinea pig, cat, dog, sheep, pig, calf and man), suggesting NPY participation in the regulation of local blood flow and thyroid activity (Grunditz et al., 1984). 
VIP is known to have powerful effects on secretion from several endocrine and exocrine glands and occurs in nerves with a ubiquitous distribution in the body (Melander et al., 1971; 1972). The thyroid of several species is supplied with VIP-containing nerve fibers that surround blood vessels and run between and along thyroid follicles; neuronal VIP participates in the regulation of thyroid hormone secretion through a mechanism that is mediated by cyclic AMP (Ahrén et al., 1980; 1982; 1991). VIP fibers have been revealed around follicles and blood vessels, and, moreover, VIP has a stimulatory effect on thyroid hormone secretion (Hedge et al., 1984; Grunditz et al., 1986).

Therefore, there is evidence that NPY and VIP act locally as autocrine or paracrine regulators.

Another regulator of thyroid activity is 5-HT. It is produced by mammalian thyroid parafollicular cells (Nunez et al., 1978) and may participate with TSH in the stimulation of follicular cells (Ericson et al., 1970; Melander, 1970; Melander et al.,1973; Tamir et al., 1996). In addition, it has been radioautographically demostrated that 5-HT is present in the colloid, and its concentration in the follicular lumen exceeds that in the follicular cells (Tamir et al., 1996).

Numerous papers exist on the immunohistochemical localization of these paracrine regulators in the thyroid gland of vertebrates, whereas only few are concerned with reptiles. Therefore, the aim of the present study was to immunohistochemically localize NPY, VIP and 5-HT in the thyroid gland of the lizard, Podarcis sicula.

\section{MATERIALS AND METHODS}

The thyroid glands of $P$. sicula were examined by immunohistochemistry with the avidin-biotin-peroxidase complex (ABC) to determine the localization of neuropeptides.

The thyroid glands were immediately removed and fixed in Bouin's solution for $24 \mathrm{~h}$, then dehydrated and embedded in paraffin wax.

Serial sections ( $5 \mu \mathrm{m}$ thick) were cut and carried through xylene and graded alcohol to water.
Slides were incubated for $30 \mathrm{~m}$ in a solution of $3 \%$ $\mathrm{H}_{2} \mathrm{O}_{2}$ in methanol $(1: 5 \mathrm{vv})$ to inhibit endogenous peroxidase activity. Then they were rinsed with distilled water and phosphate buffered saline (PBS). Non-specific binding sites for immunoglobulins were blocked by incubation in normal goat serum (NGS) for $20 \mathrm{~m}$. The sections were then incubated overnight at $4{ }^{\circ} \mathrm{C}$ in a moist chamber with anti-rabbit VIP, anti-rabbit NPY and anti-rabbit 5-HT (Biogenesis Ltd, Poole, England). Working dilutions were: 1:300 for anti-VIP, 1:100 for anti-NPY and anti-5-HT.

After washing for $10 \mathrm{~min}$ in PBS, immunohistochemical visualization was carried out using the Vecta-lab "Elite" (ABC) kit (Vector, Burlingame, $\mathrm{CA}$ ). This method utilized the avidin-biotin-peroxidase complex (ABC) procedure as follows: the sections were incubated for $30 \mathrm{~min}$ with diluted biotinylated goat anti-rabbit $\operatorname{IgG}(1: 400)$, and, after washing in PBS, they were incubated for 30 min with the Vectastain Elite ABC Reagent.

Peroxidase activity was visualized by incubating the sections with $0.01 \% \mathrm{H}_{2} \mathrm{O}_{2}$ and $0.05 \%$ diaminobenzidine tetrahydrochloride (DAB; Sigma, St. Louis, M.O.) in $0.05 \mathrm{M}$ Tris buffer, $\mathrm{pH} 7.2$ for 10 min to reveal the brown immunoreactive cells.

To confirm the specificity of the immunostaining, the following control staining procedures were carried out: 1) replacement of primary antibody with normal rabbit serum, 2) omission of primary antibody, and 3) absorption of primary antibody with homologous antigen.

\section{RESULTS}

The thyroid gland of the lizard $P$. sicula is a single discrete ribbon-like structure which transversely crosses the middle of the trachea. It is formed by follicles which are connected by an interfollicular connective tissue which contains blood vessels. A superficial connective tissue capsule envelops the gland and sends branches which form a network that surrounds the follicles.

Fig. 1 - Lizard thyroid gland. a: Control specimen; note the absence of immunoreactivity (40X); b: Immunopositive apical cells for NPY (arrowheads) and NPY+ nerve fibers (arrows) (40X); c: At higher magnification (100X), note the NPY nerve fibers (arrows); d: Immunopositive apical cells for VIP (arrowheads) and VIP antibody nerve fibers (arrows) (40X); e: Immunopositive $\mathrm{VIP}^{+}$nerve fibers around the follicles (arrows) (100X); f: Immunopositive intrafollicular colloid for 5-HT (40X); g: At higher magnification, note the 5 - $\mathrm{HT}^{+}$cytoplasmic bridge $(100 \mathrm{X})$. 

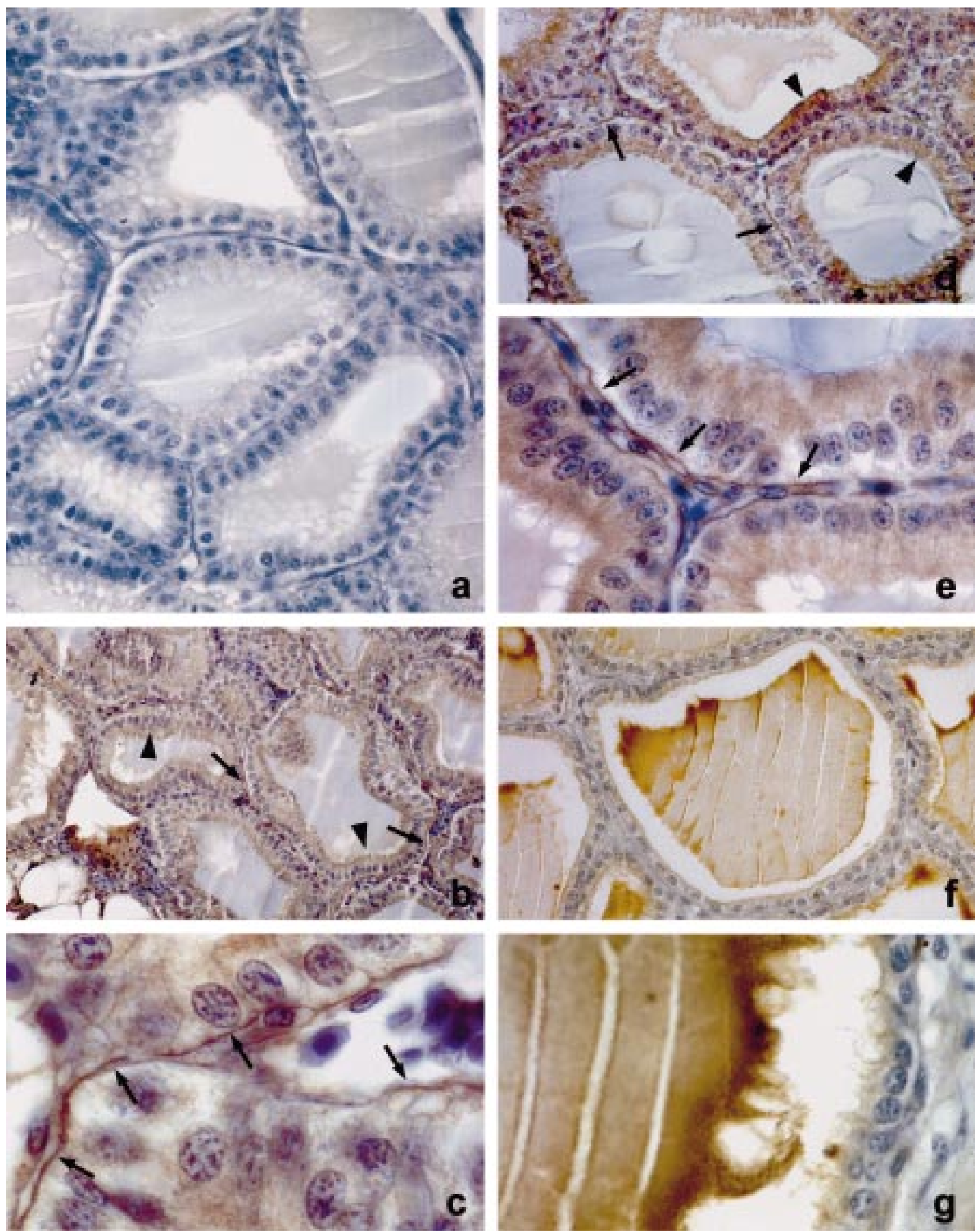
The immunostained control sections of $P$. sicula thyroid gland did not show any signal of immunoreactivity (Fig. 1a).

NPY-IR fibers were found around the follicles, and, in the cells, immunoreactivity was localized in the cellular apices (Fig. 1b,1c).

Immunoreactivity for VIP was observed in the nerve fibers surrounding blood vessels and running between and along thyroid follicles. These fibers were thin, beaded and quite uniformly distributed within the gland. VIP-immunoreactivity was also present in the apical cytoplasm of the follicular cells (Fig. 1d,1e).

5-HT-immunoreactivity was revealed in the colloid, and its concentration in the follicular lumen exceeded that in the follicular cells. The thyroid did not contain serotoninergic nerve fibers. Most follicles showed immunostaining in the cytoplasmic bridges formed between the apical portion of follicular cells and the colloid (Fig. 1f,1g).

\section{DISCUSSION}

Thyroid hormone secretion is regulated by a complex interplay of hormonal and neuronal signals, many of which are likely still to be identified. So far, three types of signals have been observed in $P$. sicula thyroid gland, storing immunoreactive VIP, NPY and 5-HT.

VIP and NPY but not serotonin fibers were observed around follicles. Immunoreactivity for VIP and NPY was also observed in the apical cytoplasm of the follicular cells, suggesting the participation of VIP and NPY in the regulation of thyroid activity. In fact, the morphological evidence that VIP and NPY fibers are distributed in the thyroid of the lizard Podarcis sicula have revealed that these two neuropeptides may be involved in the regulation of thyroid hormone secretion. Our preliminary observations have shown that in this lizard, the i.p. administration of VIP and NPY increased plasma T4 and T3 levels, demonstrating that these neuropeptides affect the secretion of thyroid hormone by acting on specific receptors.

Conversely, 5-HT was observed in the colloid and in the connection between the apical cytoplasm portion and the colloid, similarly to what has been described in most vertebrates. In mammals, sero- tonin, which is synthesized and secreted by thyroid parafollicular cells, reaches the luminal colloid and binds thyroglobulin, which is an acidic glycoprotein with an isoelectric point of 4.5 , because serotonin is highly polar at physiological $\mathrm{pH}$ (Tamir et al., 1996). The absence of serotonin immunoreactive nerve fibers around the follicles suggests that the thyroid gland of this lizard may not contain serotoninergic nerve fibers, as is instead present in most vertebrates. Our data (Sciarrillo et al., in press) have indicated that, in this lizard, intraperitoneal administration of 5-HT increases plasma T4, T3, and TSH levels and causes colloid droplet formation, indicating that 5HT is involved in the activation of thyroid follicular cells.

In conclusion, our results show the localization of these substances in the thyroid gland of the lizard $P$. sicula, suggesting a modulatory role in the control of thyroid hormone secretion for VIP, NPY and serotonin.

\section{ACKNOWLEDGEMENTS}

We wish to thank Mr. Giuseppe Falcone for typing and setting all the illustrations of the paper.

\section{REFERENCES}

Ahrén B., Alumets J., Ericson M., Fahrenkrug J., Håkanson R., Hedner P., Lorèn I., Melander A., Rerup C., and Sundler F.: VIP occurs in intrathyroidal nerves and stimulates thyroid hormone secretion. Nature 287, 343-345, 1980.

Ahrén B., Håkanson R., and Rerup C.: Vip stimulates thyroid hormone secretion: effects of other neuropeptides and $\alpha$ or $\beta$ adrenoceptor blockade. Acta Physiol. Scand. 114, 471-473, 1982.

Ahrén B.: Regulatory peptides in the thyroid gland: a review on their localization and function. Acta Endocrinol. (Copenh) 124 (3), 225-232, 1991

Ekbald E., Edvinsson L., Wahlestedt C., Uddman R., Håkanson R., and Sundler F.: Neuropeptide Y co-exists and co-operates with noradrenaline in perivascular nerve fibers. Regul. Peptides 8, 225-232, 1984.

Ericson L.E., Melander A., Owman C.H., Sundler F.: Endocytosis of thyroglobulin and release of thyroid hormone in mice by catecholamines and 5-hydroxytryptamine. Endocrinol. 87, 915-923,1970.

Grunditz T., Håkanson R., Rerup C., Sundler F., and Uddman R.: Neuropeptide Y in the thyroid gland: Neuronal localiza- 
tion and enhancement of stimulated thyroid hormone secretion. Endocrinology 115, 1537-1542, 1984.

Grunditz T., Håkanson R., Hedge G., Rerup C., Sundler F., and Uddman R.: Peptide histidine isoleucine amide stimulates thyroid hormone secretion and coexists with vasoactive intestinal polypeptide in intrathyroidal nerve fibers from laryngeal ganglia. Endocrinology 118, 783-790, 1986.

Hedge G., Huffman L., Grunditz T., and Sundler F.. Immunocytochemical studies of the peptidergic innervation of the thyroid gland in the Brattleboro rat. Endocrinology 115, 20712076, 1984.

Lundberg J.M., Terenius L., Hökfelt T., Martling C., Tatemoto K., Mutt V., Polak J., Bloom S., and Goldstein M.: Neuropeptide Y (NPY)-like immunoreactivity in peripheral noradrenergic neurons and effects of NPY on sympathetic function. Acta Physiol. Scand. 116, 477-483, 1982.

Melander A.: Interactions of adrenergic blocking drugs with the in vivo release of thyroid hormone induced by thyrotropin and the long-acting thyroid stimulator. Acta Endocrinol (Copenh) 66, 151-156, 1971 .
Melander A., Nilsson E., and Sundler F.: Sympathetic activation of thyroid hormone secretion in mice. Endocrinology 90, 194-202, 1972.

Melander A., Sundler F., and Westgreen U.: Intrathyroidal amines and the synthesis of thyroid hormone. Endocrinology 93, 193-200, 1973.

Nunez E.A., and Gershon M.D.: Formation of apical pseudopods by thyroid follicular cells: induction by thyrotropin and 5-hydroxytryptamine; antagonism by reserpine. Anat. Rec. 192, 215-224, 1978.

Sundler F., Moghimzadeh E., Håkanson R., Ekelund M., and Emson P.: Nerve fibers in the gut and pancreas of the rat displaying neuropeptide-Y immunoreactivity. Cell Tissue Res. 230, 487-492, 1983

Tamir H., Hsiung S., Liu K., Blakely R.D., Russo A.F., Clark S., Nunez E.A., and Gershon M.D.: Expression and development of a functional plasmalemmal 5-hydroxytryptamine transporter by thyroid follicular cells. Endocrinology 137 , 4475-4486, 1996. 